\title{
Petits trains départementaux : comment aborder leur histoire
}

Alain de Dieuleveult

\section{(2) OpenEdition}

\section{Journals}

Édition électronique

URL : https://journals.openedition.org/rhcf/2074

DOI : 10.4000/rhcf.2074

\section{Éditeur}

Rails \& histoire

\section{Édition imprimée}

Date de publication : 2 mai 2002

Pagination : 308-320

ISBN : 00996-9403

ISSN : 0996-9403

\section{Référence électronique}

Alain de Dieuleveult, "Petits trains départementaux : comment aborder leur histoire », Revue d'histoire des chemins de fer [En ligne], 24-25 | 2002, mis en ligne le 20 avril 2015, consulté le 22 avril 2022. URL : http://journals.openedition.org/rhcf/2074 ; DOl : https://doi.org/10.4000/rhcf.2074 


\section{Quatrième partie}

Les chemins de fer secondaires en France : histoire et patrimoine 


\section{Alain de DIEULEVEULT}

\section{Petits trains départementaux : comment aborder leur histoire}

Par une curieuse coïncidence, la naissance de ma vocation d'historien a correspondu chronologiquement, à peu de chose près, à la disparition de la plupart des réseaux de petits trains départementaux, c'est vous dire que les faits remontent - dois-je dire : hélas? - à un bon demi-siècle. En ce temps-là, les étudiants en histoire, à l'issue de la licence, étaient soumis, avant les grands concours du C.A.P.E.S. et de l'agrégation, à la réalisation d'un diplôme d'études supérieures, le D.E.S. Celui-ci, soit dit en passant, était tout autre chose que l'actuel mémoire de maitrise, tant par son ampleur même que parce que l'on devait présenter en fait deux mémoires sur des périodes d'histoire différentes.

Ayant choisi, en fonction de mes goûts de l'époque, un sujet sur le Moyen Age pour mon mémoire principal, j’étais sans idée précise pour le mémoire secondaire, quand j'eus la chance de fréquenter, aux Archives départementales de la Sarthe, un certain François Dornic, futur premier président de la nouvelle université du Maine, qui préparait alors sa thèse de doctorat, mais qui avait été aussi, en tant que conseiller général, un témoin privilégié et navré de l'agonie et de la mort des tramways de la Sarthe. Au fil de nombreuses séances passées face à face dans la salle de travail des archives, presque seuls (ce qui est devenu aujourd'hui impensable par suite de l'invasion des généalogistes amateurs), il me convainquit d'en écrire l'histoire et n'eut d'ailleurs pas trop de mal à le faire, car j'étais à la fois un amoureux de toujours des chemins de fer et un ancien usager assidu de plusieurs lignes des dits petits trains.

Cependant, quand j'allai présenter mon sujet à mon professeur de l'université de Rennes, Henri Fréville, il me répondit tout crûment : « Mais ce n'est pas de l'histoire!» Vous voyez, Mesdames et Messieurs, vous qui êtes tout convaincus d'avance, qu'il y avait du chemin à parcourir pour donner ses lettres de noblesse à l'histoire des petits trains départementaux, c'est-à-dire surtout, en ne se contentant pas de comptabiliser bêtement rails, locomotives et wagons, d'insérer pleinement cette histoire dans la grande histoire, avec toutes les dimensions politiques, économiques, sociales et culturelles qu'elle implique. 
Ce chemin, autrefois, nous n'avons pas été nombreux à l'emprunter et si, de nos jours, le travail accompli par diverses associations et dans plusieurs revues de caractère technique a été considérable, les études proprement historiques concernant globalement tel ou tel réseau départemental de petits trains demeurent rares. Je n'en suis que plus enclin à faire au passage la publicité de la collection « Le siècle des petits trains » dont les éditions Cénomane poursuivent depuis plus d'une quinzaine d'années la publication, avec aujourd'hui quinze titres parus, couvrant la quasi-totalité du grand Ouest, plus des antennes vers le Jura, la Savoie et même l'île de la Réunion, collection que je dirige et au sein de laquelle je me permets de préciser que j’ai rédigé personnellement sept ouvrages, du premier, la Sarthe, en 1985, au dernier-né, le Loir-etCher, en 2001, et que j'en prépare actuellement un huitième. D'ailleurs, cela étant, mes auditeurs n'auront pas lieu de s'étonner que, au cours de mon propos, les exemples concrets venant à l'appui de ma démonstration soient tirés de ce même grand Ouest.

\section{Comment aborder I'histoire des petits trains départementaux ?}

Mais d'abord, de quoi s'agit-il ? " Petits trains départementaux », l'appellation dit tout !

Ce sont des trains : historiquement, ils figurent comme un épisode (voire un avatar) de l'aventure générale des chemins de fer au $\mathrm{XIX}^{\mathrm{e}}$ siècle et au début du $\mathrm{XX}^{\mathrm{e}}$ siècle ; techniquement, ce sont bien des convois de véhicules tractés par un engin moteur sur des rails posés, par l'intermédiaire de traverses, sur une voie particulière (en site propre ou en accotement des routes).

Ils sont petits et même... tout y est petit : les voies (avec un écartement entre les rails de $0,60 \mathrm{~m}$ à $1 \mathrm{~m}$, rarement davantage), les engins tracteurs (locomotives, plus tard automotrices), les voitures et wagons, les gares, etc., mais aussi la vitesse, la capacité de transport et... les prétentions.

C'est donc assez dire que les louanges souvent dithyrambiques adressées de nos jours à ce moyen de transport d'un autre âge, respectables si elles sont de nature sentimentale, deviennent démesurées et ridicules dès lors qu'il s'agit d'en apprécier les performances techniques ou commerciales.

Ils sont enfin départementaux, mais ceci nécessite une explication historique un peu plus approfondie.

A partir de la loi du 11 juin 1842 s'était édifié en France un réseau ferroviaire national disposé principalement en étoile autour de Paris et dont les lignes furent exploitées par la suite par de grandes compagnies capitalistes, Ouest, P.O., P.L.M., etc. Cependant, à partir du moment où 
il s'agit de réaliser des liaisons de plus en plus secondaires, donc de moins en moins rentables, ces grandes compagnies et les investisseurs furent beaucoup moins intéressés. On eut alors l'idée de génie, à la fin du Second Empire, de transférer une partie des responsabilités de l'État aux départements en matière de construction, d'exploitation et de contrôle des voies ferrées : c'est l'objet de la loi du 12 juillet 1865, véritable acte de naissance des «petits trains » (plus tard complétée par celle du 11 juin 1880, qui clarifiera définitivement la situation). Loi entrainant une double conséquence : d'une part le rôle prépondérant des institutions départementales, conseil général, préfet et fonctionnaires relevant de son autorité, d'autre part le caractère presque exclusivement départemental des réseaux qui vont se constituer.

Administrativement, le pouvoir de décision et la tutelle des réseaux appartiennent aux dites institutions ; financièrement, le département recourt à des emprunts, couverts par l'imposition de centimes additionnels, qui ont grevé le budget départemental lourdement, pour longtemps et même, dans certains cas, au-delà de la durée des réseaux construits ; il a pu même arriver, situation extrême et aberrante que j’ai expressément vérifiée, par exemple dans la Sarthe, que les contribuables dussent continuer à payer (et les rentiers à toucher des intérêts) pour des lignes qui ne furent jamais construites ; techniquement, enfin, la construction et la gestion des réseaux sont concédées par le département, en règle générale, à des sociétés privées, mais il en conserve le contrôle.

Enfin, disons plutôt qu'il s'ingénie à le conserver, tant il lui a été difficile, de s'assurer, pendant la durée quasi totale du « siècle des petits trains », la maitrise sur des compagnies concessionnaires. Parmi cellesci, en effet, non seulement je n'ai jamais trouvé, au cours de mes recherches, le partenaire idéal, mais encore je n'ai même jamais rencontré d'exploitant avec lequel les relations n'aient été difficiles et le torchon n'ait fini par brûler. Absence de souci de l'intérêt général, défaut d'entretien de l'outil de travail, mépris de l'usager, manque de considération à l'égard du personnel, escamotage des responsabilités, notamment en cas d'accidents, enfin roublardise comptable faisant apparaitre un déficit chronique (" délit d'habitude » des compagnies, dira un préfet de Loire-Inférieure), tandis que de solides dividendes faisaient le bonheur des actionnaires, la cause parait définitivement entendue.

Et pourtant, dans les écrits techniques spécialisés, je crois bien n'avoir jamais lu quoi que ce soit qui mette en cause la compétence ou les agissements de Mesdames les compagnies, envers lesquelles on fait en général preuve, au contraire, d'un respect qui, ne pouvant être mis au compte de la complicité, confine à la naïveté. 
Quant à la géographie des réseaux, on peut formuler deux remarques : nombre d'entre eux, à l'imitation du réseau national, se sont constitués en étoile autour du chef-lieu de département (tels les exemples remarquables de la Sarthe ou de l'Ille-et-Vilaine, mais aussi les contre-exemples flagrants de l'Indre-et-Loire et du Loir-et-Cher) ; les frontières interdépartementales sont demeurées, dans la plupart des cas, rigoureusement imperméables (le Blanc-Argent que vous parcourrez prochainement étant l'exception qui confirme la règle), et ce ne fut pas l'une des moindre tares du système.

«Le siècle des petits trains » se situe en gros entre la fin du Second Empire et les lendemains de la Seconde Guerre mondiale. Les réseaux ferrés d'intérêt local ont donc entièrement disparu depuis maintenant au moins un demi-siècle et les compagnies qui les exploitaient ont été dissoutes ou se sont reconverties de longue date dans d'autres activités, en particulier, bien sûr, dans le transport routier (à l'instar, par exemple, de la société Verney dont les cars ont remplacé les trains dans nombre de départements). C'est dire que c'est avant tout un travail d'historien qui est demandé à un auteur de monographie et que sa première démarche doit être d'entrer en relations avec le dépositaire de la grande majorité des documents susceptibles de l'intéresser, à savoir les archives du département concerné.

Les Archives départementales conservent trois catégories de documents qui sont pour nous de toute première importance :

\section{D’abord et avant tout les recueils imprimés du conseil général}

Tout dépôt d'archives possède la collection complète de ces recueils, où l'on trouve les rapports des préfets et des chefs de services (dont les Ponts et Chaussées, chargés de la préparation, puis du contrôle des voies ferrées d'intérêt local), les rapports des commissions de l'assemblée, le compte rendu des débats, les vœux et les réponses qui y sont apportées, etc.

Ces recueils étaient remarquablement tenus, du moins jusqu'à la Seconde Guerre mondiale, et comme les petits trains ne sont pas loin d'y occuper souvent la première place en intérêt et... en nombre de pages (parfois, dans l'Ouest, en concurrence avec la grave question des bouilleurs de cru...), ils constituent une source de documentation absolument indispensable, dont on peut dire qu'à la limite le chercheur pressé pourrait se contenter. Je citerai un cas que j'ai vécu concrètement, celui de la Manche, dont le dépôt d'archives avait été détruit dans les bombardements de Saint-Lô en 1944-1945 et dont presque seuls les recueils imprimés, patiemment rassemblés par un conservateur d'une 
compétence et d'une conscience en tous points exemplaires, Yves Nédellec, nous ont permis de réaliser un ouvrage aussi substantiel, sinon plus, que les autres volumes de la collection.

La consultation des recueils imprimés peut paraitre fastidieuse, mais elle est grandement facilitée par les tables récapitulatives qui figurent à la fin de la partie relative à chaque session du conseil général ; sachons qu'il y avait d'habitude deux sessions ordinaires par an, parfois complétées d'une ou plusieurs sessions extraordinaires qui, comme par hasard, étaient souvent consacrées aux petits trains et qu'il importe donc de ne pas oublier au passage.

L'usager constatera en outre que, parfois, ces recueils n'ont jamais été consultés avant lui : quand il lui arrive de travailler avec le coupe-papier, il a encore plus le sentiment de faire œuvre parfaitement originale, tout en se demandant comment peuvent bien procéder les soi-disant experts qui meublent de leur torrent de précisions techniques et de leur prose... approximative certaines publications spécialisées, sans parler de ceux qui, prétendant écrire un ouvrage sur les petits trains de tel département, sont purement et simplement inconnus au fichier des archives de son chef-lieu...

\section{En deuxième lieu, le fonds dit des V.F.I.L., autrement dit des voies ferrées d'intérêt local}

Les Archives départementales conservent, dans ce fonds, des monceaux de documents relatifs à l'histoire des petits trains, souvent des centaines de liasses contenant chacune des dizaines ou des centaines de documents. Ils relèvent principalement de la série $5 \mathrm{~S}$, qui est aujourd'hui en général soigneusement classée, mais que, dans mes premières années de recherches, $j$ 'ai connue dans un état d'anarchie qui en dit long sur le peu d'intérêt que ce type de documents suscitait alors. Notons que des fonds appartenant à d'autres séries peuvent également contenir des documents se rapportant aux voies ferrées d'intérêt local, en particulier la série J regroupant des fonds privés.

Ce patrimoine documentaire est quasi exclusivement d'origine publique (dépôt de la préfecture, des Ponts et Chaussées et aujourd'hui de l'Equipement, du conseil général) : en effet, sauf de très rares exceptions auxquelles il faut rendre hommage (je pense en particulier au Loir-et-Cher), les compagnies concessionnaires, à la fin de l'exploitation, ont préféré détruire leurs archives, dont il ne subsiste aujourd'hui que des épaves, plutôt que de les remettre à l'autorité de tutelle. Voilà une belle preuve posthume de leur mépris de l'intérêt général et de leur volonté d'emporter dans la tombe des secrets que l'on se laissera facilement aller à qualifier d'inavouables... 
Il va sans dire qu'il est absolument impossible de consulter la totalité de la série $5 \mathrm{~S}$, il y faudrait des années. D’ailleurs, nombre des documents qu'elle contient ne font figure, en quelque sorte, que de brouillon des recueils du conseil général, alors autant recourir directement au propre, en espérant qu'il ne soit pas trop résumé.

\section{En troisième lieu, les collections de la presse locale}

Autrefois, tout département possédait un ou plusieurs journaux locaux, en général hebdomadaires, qui, étant donné le caractère le plus souvent « rural » des petits trains, s'y intéressaient davantage que les grands journaux régionaux et, à plus forte raison, nationaux.

La consultation des collections de ces journaux locaux, conservées aux Archives (et parfois dans les bibliothèques municipales), est indispensable : ils se font l'écho des débats autour des petits trains, relatent les inaugurations et fermetures de lignes, les accidents, les conflits sociaux, accueillent les communiqués des élus et les plaintes des usagers, reproduisent les horaires, tarifs et autres annonces, etc.

\section{Les trois catégories de documents précitées sont loin de constituer les seules ressources des Archives départementales}

\section{Citons encore :}

- Les résultats des recensements de population. Ils sont regroupés soit en fascicules édités par les observatoires régionaux de l'I.N.S.E.E., soit sous forme de tableaux réalisés par les Archives elles-mêmes. L'exploitation de ces tableaux permet de mieux mesurer l'impact humain et économique des petits trains.

- Les listes de personnages importants, préfets, ingénieurs en chef des Ponts et Chaussées, parlementaires, conseillers généraux, etc. Leur nomenclature va, suivant les départements, des énumérations sommaires aux ouvrages biographiques complets. Les conseillers généraux, surtout, ayant été les grands acteurs de la réalisation des réseaux ferrés locaux, il serait impardonnable de ne pas les connaitre ni les citer comme de négliger leurs options politiques ; pourtant, j’ai bien souvent parcouru des articles de publications spécialisées sur les chemins de fer départementaux où l'on ne trouvait nommé aucun conseiller général, aucun préfet, aucun ingénieur en chef des Ponts et Chaussées ni cité aucun extrait des débats de l'assemblée départementale. Il m'est même arrivé de lire, à propos du Loir-et-Cher, que les débats de son conseil général relatifs aux petits trains avaient été houleux, alors qu'au cours de mes recherches je n'ai jamais rencontré de département où le consensus ait été plus parfait. 
- L'Annuaire qui se publiait autrefois dans certains départements, sorte de bottin local contenant toutes sortes de renseignements pratiques, noms des personnalités importantes, population des communes, horaires et tarifs des transports, publicités commerciales, etc.

- Les ouvrages d'histoire locale, dont la bibliothèque des Archives est en général fournie (et, à son défaut, la bibliothèque municipale du cheflieu), au premier rang desquels il faut citer, quand ils existent, l'histoire du département dans la collection des Editions Bordessoules et les monographies des Editions Privat sur telle ou telle ville et sa région (collections qui sont l'une et l'autre en cours de réalisation).

- La collection du Bulletin des Lois, qui a d'ailleurs l'avantage de pouvoir être consultée n'importe où et non forcément aux Archives du département concerné. C'est une source capitale, car elle publie intégralement les déclarations d'utilité publique des lignes et réseaux de petits trains et le texte des conventions et cahiers des charges, documents contenant des informations abondantes et précises sur les caractéristiques techniques, le tracé des lignes, les tarifs, etc. (en ce qui concerne les tarifs, il faut se rappeler que l'on est, du moins jusqu'à la Grande Guerre, dans une période de stabilité monétaire parfaite, qui les a rendus intangibles durant de très nombreuses années). Signalons en outre que, jusqu'à la même Grande Guerre, le Journal officiel publiait en annexe les résultats d'exploitation de l'ensemble des chemins de fer d'intérêt local et tramways, ce qui permet de dresser un tableau des recettes et dépenses du réseau étudié, éléments que l'on ne trouve pas toujours dans les recueils imprimés du conseil général ni dans le fonds des V.F.I.L.

Quant aux illustrations, on pourra les trouver dans le fonds des V.F.I.L. (affiches, tableaux horaires, cartes de lignes, plans de gares, graphiques d'exploitation, etc.) mais, bien entendu, il faut recourir principalement aux cartes postales. La raison en est simple : l'âge d'or de la carte postale correspond à peu près exactement à celui des petits trains, et ceux-ci ont même été, à la fin du XIXe siècle et au début du $\mathrm{XX}^{\mathrm{e}}$, le sujet de prédilection des photographes. D'où une double conséquence : d'une part, il est indispensable d'y recourir massivement pour étayer en quelque sorte de preuves en images l'histoire des petits trains (et il n'y manquera, excusez du peu, que le mouvement, le bruit et l'odeur !) ; d'autre part, hélas, ces documents atteignent aujourd'hui des cotes considérables, les plus élevées sans doute sur le marché de la carte postale. Alors, comment se les procurer?

On obtiendra d'ordinaire facilement le droit de reproduire gratuitement, moyennant mention d'origine, les cartes postales appartenant aux Archives départementales, voire à des bibliothèques, musées, etc., 
mais l'importance de ces fonds, dont la constitution est en général récente, est très variable, allant de l'abondance à l'indigence. Il faut donc bien, dans la plupart des cas, faire appel aux particuliers.

L'accès au milieu si spécial des collectionneurs de cartes postales est très délicat : il faut d'abord les débusquer et vaincre leur méfiance, d'autant que la législation sur la propriété et les droits de reproduction de ce type de documents est assez ambiguë.

L'idéal est évidemment de tomber sur « le » collectionneur qui, passionné à la fois de son département et des petits trains, possède pratiquement tout ce qui est paru, et d'en obtenir l'autorisation de reproduire ses documents, en négociant les conditions de cette reproduction. Disons toutefois tout net qu'il vaut mieux renoncer - et chercher ailleurs - que de céder à des prétentions financières qui peuvent s'avérer aussi exorbitantes qu'infondées. Dans la plupart des cas, en fait, on a affaire à une multitude de collectionneurs possédant chacun quelques cartes sur le sujet, avec lesquels il faudra négocier au coup par coup et, en fait de coups, surtout à coup de sourires.

Pas d'étude historique sans recherche sur le terrain. Le terrain, c'est le département, avec lequel le chercheur n'est probablement pas familiarisé au départ, même si, à l'arrivée, il aura fini par le sillonner en tous sens et le connaitre comme sa poche! A cet égard, déclarons qu'il ne faut pas avoir de complexe vis-à-vis des «autorités » historiques locales reconnues : à force de recherches, d'observations et de contacts, on finira par en savoir bien plus qu'elles - et surtout le savoir bien mieux - dans le domaine qui nous intéresse.

En outre, si l'histoire des chemins de fer en général a peu attiré les historiens, c'est encore plus vrai au niveau départemental. Dans la Sarthe, par exemple, si l'on dépouille minutieusement, pour les XIX et $\mathrm{XX}^{\mathrm{e}}$ siècles, la collection des revues savantes publiées par les trois sociétés érudites locales, Société historique et archéologique du Maine, Société historique de la province du Maine et Société d'agriculture, sciences et arts de la Sarthe, on n'y trouve pas le moindre article sur les chemins de fer et, a fortiori sur les petits trains, mis à part, en 1963, c'est-à-dire treize ans après la soutenance, le texte intégral de mon mémoire universitaire sur les tramways du département, sauvé d'un oubli total grâce à un heureux concours de circonstances.

Pour une première approche du département, en dehors du Bordessoules, il est facile de recourir - d'autant que, chronologiquement, ces sources sont contemporaines de l'histoire des petits trains - à la notice contenue dans la Grande Encyclopédie du XIX siècle (dite "Encyclopédie Berthelot»), aux petites monographies 
d'Adolphe Joanne, récemment rééditées pour la plupart des départements, et aux guides Joanne (ancêtres des Guides bleus Hachette) dans des éditions de la fin du XIX ${ }^{\mathrm{e}}$ siècle ou du début du XXe

Une autre approche qui, celle-ci, a l'intérêt de contenir de nombreux passages où sont évoqués les petits trains, est fournie par le célèbre Voyage en France d'Ardouin-Dumazet qui, en une soixantaine de volumes échelonnés entre 1890 et la Première Guerre mondiale, a décrit minutieusement les régions qu'il a parcourues à pied, en voiture à cheval et en chemin de fer ; source d'autant plus intéressante qu'il peut exister plusieurs éditions successives du même ouvrage et que l'on peut ainsi mesurer la progression de la construction des petits trains et de leur impact sur la vie économique, sociale et même culturelle de la région considérée.

Un simple échantillon d'Ardouin-Dumazet, relatif au Morbihan, à titre de preuve de pertinence : « Je crois bien, écrit-il, qu'aucune autre population ne se déplace aussi facilement que le peuple breton, accoutumé depuis tant d'années à fréquenter les pèlerinages et les pardons. Aussi les voies ferrées dont on couvre toute la presqu'île d'Armorique donnent-elles d'excellents résultats. » Mais le même auteur, non dépourvu de causticité, poursuit : «Ces gens, hélas! restent non moins fidèles aux habitudes d'ivrognerie ; s'il est trop matin pour que j'aie l'affligeant spectacle d'une fin de dimanche breton, les quais de la petite gare me montrent de grands fûts d'alcool portant la marque d'une distillerie de Denain, dans le Nord, eau-de-vie de grain à bas prix qui a de si funestes effets sur la race. » Passons pudiquement...

Nul évidemment ne peut avoir la prétention d'être tout à fait un pionnier. Il faut par conséquent recenser tout ce qui a pu être publié sur le thème proprement ferroviaire. Dans ce domaine, il convient de faire une place tout à fait à part au travail d'un remarquable connaisseur national des petits trains, le regretté Henri Domengie : dans les cinq volumes des Petits trains de jadis, que lui et ses continuateurs ont publié aux Éditions du Cabri entre 1983 et 1995, sont passés en revue tous les réseaux locaux, département par département, avec étude historique sommaire et tableau récapitulatif des lignes ayant existé ; de plus, le volume consacré au Sud-Est contient une introduction générale d'une dizaine de pages dont la lecture est indispensable.

Les diverses revues ferroviaires, que la plupart d'entre vous connait sans doute fort bien, ont publié de nombreux articles sur les réseaux départementaux : leurs informations de caractère technique (voies, bâtiments, matériel roulant, etc.) sont extrêmement utiles, mais leur apport à une véritable histoire des petits trains (une histoire " historienne", 
comme se plaît à le répéter mon éditeur) est pratiquement inexistant. Il en est de même pour la grande majorité des ouvrages qui ont pu être réalisés sur tel ou tel réseau départemental par diverses maisons régionales, ouvrages qui se réduisent dans la plupart des cas à une chatoyante collection d'images.

Voici à présent, simplement quelques pistes pour la recherche proprement dite sur le terrain :

- le contact avec les associations d'anciens des petits trains, de "mordus» des questions ferroviaires (ceux que les historiens surnomment " ferrovipathes », sans méchanceté d'ailleurs, puisque, pour ma part, j’ai cosigné plusieurs ouvrages avec l'un d'entre eux et des plus connus et respectés, l'Angevin Michel Harouy, qui anime depuis des années une association exemplaire entre toutes, Les Amis du Petit Anjou), de restaurateurs de matériels anciens et de modélistes, d'animateurs de chemins de fer touristiques, etc. ;

- la rencontre avec des témoins du temps des petits trains, agents des compagnies ou usagers ;

- la recherche des vestiges du réseau disparu, tracés de lignes, ouvrages d'art, gares, matériel, etc., dont il nous faut bien déplorer qu'après la Seconde Guerre mondiale, dans un souci, qu'on peut évidemment comprendre, de tourner délibérément le dos au passé, on se soit semble-t-il véritablement acharné à les faire disparaitre.

Dans cette quête des vestiges, il faut évidemment suivre les anciennes lignes ; on y est heureusement aidé par des cartes contemporaines de l'existence des petits trains, soit les plus vieilles cartes Michelin au 1/200 $000^{e}$, où le tracé est en général suffisamment précis et toutes les gares et haltes indiquées, soit, pour plus de détails, les cartes d'état-major.

Tout ce travail sur le terrain n'est pas évident : les associations, quand elles existent, n'ont pas toujours pignon sur rue et la rareté de leurs membres en entraîne la confidentialité ; les «mordus » sont gens très exclusifs, dont il faut gagner la confiance, et il leur arrive d'avoir le défaut bien humain de prétendre tout savoir et surtout de dénier à quiconque le droit de s'introduire dans leur domaine soi-disant réservé ; les témoins sont de plus en plus rares et âgés, partant de moins en moins fiables, et parfois seule la littérature permet de les suppléer (nous pensons à un Guéhenno en Ille-et-Vilaine, à un Jakez Hélias en Finistère) ; les vestiges existent en quantité très inégale (surabondants par exemple en Indre-et-Loire ou en Finistère, indigents dans la Sarthe) et en ordre dispersé ; ils sont souvent cachés ou défigurés par une transformation moderne, leur identification même peut être difficile quand on s'adresse à des personnes des jeunes générations, qui ne les connaissent que par ouï-dire. 
Une anecdote à ce sujet. Quand on est familier des petits trains, on finit par être conditionné à en détecter les traces au hasard de ses voyages. Me trouvant à Moëlan-sur-Mer en cours de préparation de l'ouvrage sur le Finistère, je m’adressai à un couple âgé pour lui demander où se trouvait l'ancienne gare : «Ah! mon pauvre Monsieur, il y a bien longtemps qu'elle a été démolie.» Voulant pourtant en avoir le cœur net, je continuai à déambuler dans la localité et finis par tomber nez à nez avec un bâtiment qui avait toute la tête de l'emploi. Me voyant armé d'un appareil photo, le propriétaire sortit sur le seuil avec un air pas très aimable mais, dès que je lui posai la question fatidique, sa physionomie changea complètement : oui, il s'agit bien de l'ancienne gare des petits trains. Alors il m'entraina dans son arrière-boutique, me montra divers documents et... j'eus beaucoup de mal à m’en débarrasser.

Histoire « historienne », avons-nous dit. Foin, cependant, de toute prétention érudite. Pour rendre attrayante l'histoire des petits trains aux yeux de ce qu'il est convenu d'appeler le grand public, il faut avant tout, en toute simplicité, qu'elle soit lisible, tant par le texte que par l'illustration. Il est parfaitement possible, en effet, de concilier un travail irréprochable sur le plan scientifique, sans tomber pour autant dans une sèche nomenclature, et les éléments qui font aussi d'un ouvrage un roman d'aventures, un bel album d'images et, pourquoi pas, un poème où palpite l'âme des gens d'autrefois et celle du personnage central qui était si vivant à sa façon et dont les plus âgés d'entre nous gardent la nostalgie.

Et précisément, pour ne pas finir sur une note trop prétentieusement universitaire, une anecdote et un poème.

\section{Anecdote}

Nous sommes sur la ligne Le Grand-Lucé-Le Mans des tramways de la Sarthe, au temps de leur splendeur... en sursis, c'est-à-dire pendant la Seconde Guerre mondiale. Le train suit l'accotement d'une route départementale, mais, aux environs de Parigné-l'Evêque, il existe une section à forte pente qu'il aurait bien du mal à gravir ; il fait donc un grand crochet sur la droite à travers bois et réapparait au sommet de la côte. Alors le jeu consiste, pour mes frères et moi, à sauter de la plateforme en marche à l'amorce de la déviation et à courir à toutes jambes le long de la route pour arriver en haut avant le train, ce qui ne manque jamais. 
De cette anecdote, j’atteste la vérité. En revanche, que penser de la fameuse histoire du train qui ne réussit pas à monter une côte et que les voyageurs sont invités à pousser. Cette histoire, je l'ai entendue dans tous les départements où j’ai travaillé, sans aucune exception, elle fait partie intégrante de la légende du petit train et s'est sans doute répandue, à partir du lieu où elle s'est peut-être produite une fois, sur tout le territoire national, tant il est vrai que, comme dit le proverbe, « on ne prête qu'aux riches ».

\section{Poème}

Au long du talus verdissant,

Le vieux petit train hors d'haleine

Va, vient, toujours monte et descend

Entre la colline et la plaine.

Sitôt qu'il arrive, il repart

En soufflant très haut sa fumée.

Toujours un peu plus en retard...

Il n'a pas d'heure accoutumée.

Il va, comme les promeneurs,

Il flâne et repasse avec joie

Pour mieux vous faire les honneurs

D’un verger qui borde la voie.

Les jardins laissent retomber

Sur lui leurs roses entrouvertes

Qu'on s'égratigne à dérober

A la pointe des branches vertes.

Tranquille aux rameaux des buissons

Où son nid printanier s'accroche,

L'oiseau redouble ses chansons

Au lieu de fuir quand il approche. 
Rien ne daigne plus s'effrayer,

Le feuillage à peine frissonne,

Car c'est un passant familier

Qui ne dérange plus personne.

Poème anonyme trouvé au dos d'une carte postale, publié in Alain Cornu, Petits trains des Côtes-du-Nord, Le Mans, Editions Cénomane, 1987. 